\title{
PROPRIEDADES MECÂNICAS E SOLUBILIDADE DE FILMES DE GELATINA RETICULADOS COM TRANSGLUTAMINASE
}

\author{
R. K. NISHIHORA ${ }^{1}$, E. NIEHUES ${ }^{1}$ e M. G. N. QUADRI ${ }^{1 *}$ \\ ${ }^{1}$ Universidade Federal de Santa Catarina, Departamento de Engenharia Química e Engenharia de \\ Alimentos \\ E-mail para contato: mara@enq.ufsc.br*
}

\begin{abstract}
RESUMO - Filmes biopoliméricos têm contribuído com o setor de embalagens ecológicas e biodegradáveis. Contudo, aqueles de origem proteica são pouco aplicados nessa área devido ao caráter hidrofílico desse material. No presente trabalho, foram produzidos filmes de gelatina suína tipo A, utilizando a enzima transglutaminase (TGase) como agente reticulante. Através de um planejamento experimental composto central, foram determinados os efeitos da concentração de gelatina e quantidade de enzima na solubilidade de filmes em meio aquoso bem como suas características mecânicas: a tensão máxima de ruptura $(\sigma)$, módulo de Young $\left(\mathrm{E}_{0}\right)$ e alongamento $(\varepsilon)$. Verificou-se que a solubilidade foi reduzida de maneira significativa apenas para no nível máximo de TGase $(0,231 \% \mathrm{~m} / \mathrm{V})$. As propriedades mecânicas tiveram comportamento similar à solubilidade, pois houve redução da tensão de ruptura e alongamento com o aumento da concentração de ambos, sendo isso indicativo do aumento de fragilidade do filme devido ao efeito de reticulação da cadeia polimérica. No entanto, o módulo de Young teve maiores valores em níveis baixos de concentração de gelatina e $0,2 \%$ de transglutaminase.
\end{abstract}

\section{INTRODUÇÃO}

Os biopolímeros, assim como os polímeros, são macromoléculas constituídas por moléculas menores, os monômeros, isto é, a unidade de repetição que forma o polímero ou biopolímero (Saldivar-Guerra e Vivaldo-Lima, 2013). Nesse contexto, as proteínas tem atraído grande atenção nas últimas décadas como possíveis fontes para o desenvolvimento de novos materiais de constituição polimérica devido à sua estrutura macromolecular altamente polar e reativa (Ebnesajjad, 2012).

Materiais proteicos, como é o caso da gelatina, tem ampla aplicação em recobrimento de superfícies bem como na produção de filmes comestíveis, pois a película formada tem capacidade de preservar as características organolépticas dos alimentos (Nussinovitch, 2009). Isso se deve ao fato de que polímeros naturais como as proteínas fornecem boas barreiras para gases como o $\mathrm{O}_{2} \mathrm{e}$ o $\mathrm{CO}_{2}$, contudo são bastante sensíveis a presença de água (Baldwin et al., 1995). Contudo, uma das formas de reduzir a permeabilidade e a solubilidade em água de filmes de gelatina é através do processo de reticulação ou também denominado "crosslinking", o qual promove a união de duas ou mais cadeias poliméricas por meio de uma ponte de átomos entre elas, todos ligados por 
ligação covalente (Canevarolo, 2006).

Dentre os diferentes meios de reticulação existentes, há diversos estudos sendo realizados no processo de reticulação da gelatina por via enzimática, no qual a enzima transglutaminase é foco de alguns trabalhos descritos na literatura, pois esta é capaz de catalisar reações de transferência de grupos acil formando ligações cruzadas intra e intermoleculares em proteínas, peptídeos e várias aminas primárias, principalmente através de ligações covalentes entre resíduos de glutamina e lisina (Macedo e Sato, 2005). Esse tipo de tratamento além de promover diminuição do caráter hidrofílico de filmes de gelatina, também altera as propriedades mecânicas do material (Bhattacharya et al, 2008).

Portanto, o presente trabalho objetivou avaliar através de um planejamento experimental composto central os efeitos da concentração de gelatina e quantidade de enzima na solubilidade de filmes em meio aquoso bem como suas características mecânicas: tensão máxima de ruptura, módulo de Young e alongamento.

\section{MATERIAIS E MÉTODOS}

\subsection{Materiais}

Gelatina suína tipo A (bloom 280 e Mesh 30), gentilmente cedida pela Gelnex The Gelatin Specialists (Itá, SC, Brasil). Enzima Transglutaminase ACTIVA WM ${ }^{\circledR}$ (obtida do Streptomyces mobaraense) doada pela Ajinomoto do Brasil Indústria e Comércio de Alimentos Ltda. (São Paulo, SP, Brasil).

\subsection{Planejamento experimental}

Realizou-se um planejamento experimental composto central onde os fatores estudados correspondem às concentrações de gelatina e enzima, como mostrado na Tabela 1.

Foram preparados também filmes de gelatina com as mesmas concentrações descritas na Tabela 1, porém sem a adição da enzima. As respostas avaliadas foram: percentual de solubilidade, e as propriedades mecânicas (módulo de Young, alongamento, tensão máxima de ruptura). Os dados foram analisados estatisticamente no software Statsoft STATISTICA ${ }^{\circledR} 10$, através da análise de variância (ANOVA) com nível de significância a 5\%.

\subsection{Preparo dos filmes}

Os filmes de gelatina foram produzidos em diferentes concentrações com diferentes quantidades de enzima, conforme descrito no planejamento experimental. O procedimento de preparo iniciava-se com a solubilização da gelatina na água destilada $(40 \mathrm{~mL})$ utilizando agitador magnético com aquecimento, no qual a solução era aquecida a aproximadamente $60^{\circ} \mathrm{C}$ durante 10min, facilitando a solubilização completa da proteína em questão. Em seguida a solução era mantida sobre agitação leve a $50^{\circ} \mathrm{C}$ por $30 \mathrm{~min}$, sendo que após esse período adicionava-se a 
enzima mantendo a temperatura na faixa descrita e agitação por 1h. A solução filmogênica era transferida para uma placa quadrada de politetrafluoretileno (PTFE) de $12 \mathrm{~cm}$ de largura e comprimento, e 1,0 cm de altura, que era acondicionada em estufa com circulação de ar forçada a $30^{\circ} \mathrm{C}$ por $24 \mathrm{~h}$.

Tabela 1 - Planejamento experimental composto central

\begin{tabular}{|c|c|c|c|c|c|}
\hline \multirow{2}{*}{ Ensaio } & \multirow{2}{*}{ Ordem } & \multicolumn{2}{|c|}{ Variáveis Codificadas } & \multicolumn{2}{|c|}{ Valores reais } \\
\hline & & $\mathbf{X}$ & $\mathbf{Y}$ & [Gelatina] $\mathrm{mg} / \mathrm{mL}$ & TGase $\%(\mathrm{~m} / \mathrm{V})^{*}$ \\
\hline 1 & 3 & -1 & -1 & 80,00 & 0,050 \\
\hline 2 & 7 & +1 & -1 & 180,00 & 0,050 \\
\hline 3 & 1 & -1 & +1 & 80,00 & 0,200 \\
\hline 4 & 5 & +1 & +1 & 180,00 & 0,200 \\
\hline 5 & 2 & $-\alpha$ & 0 & 59,29 & 0,125 \\
\hline 6 & 10 & $+\alpha$ & 0 & 200,71 & 0,125 \\
\hline 7 & 9 & 0 & $-\alpha$ & 130,00 & 0,020 \\
\hline 8 & 6 & 0 & $+\alpha$ & 130,00 & 0,231 \\
\hline 9 & 4 & 0 & 0 & 130,00 & 0,125 \\
\hline 10 & 8 & 0 & 0 & 130,00 & 0,125 \\
\hline 11 & 12 & 0 & 0 & 130,00 & 0,125 \\
\hline 12 & 11 & 0 & 0 & 130,00 & 0,125 \\
\hline
\end{tabular}

\subsection{Teste de solubilidade}

A medida do percentual de material solúvel em água foi realizada conforme método descrito por Cuq et al.(1997) com pequenas adaptações propostas por Carvalho e Grosso (2006). Amostras foram recortadas no formato de quadrados com $2 \mathrm{~cm}$ de largura e comprimento e realizadas as medidas de massa inicial em função da umidade da amostra. Posteriormente, imergidas em $50 \mathrm{~mL}$ de água destila e submetidas a banho termostático com agitação suave durante $24 \mathrm{~h}$ a $25^{\circ} \mathrm{C}$. Assim que transcorrido $24 \mathrm{~h}$ de imersão, as amostras foram retiradas da água e submetidas a secagem em estufa de circulação forçada de ar, por $24 \mathrm{~h}$ a $105^{\circ} \mathrm{C}$.

\subsection{Propriedades mecânicas}

A determinação das propriedades mecânicas: tensão máxima de ruptura, alongamento máximo e módulo de Young; consistem de média de três repetições. Os testes de tração foram realizados em texturômetro (TA. HD. Plus, Stable Micro Systems), com carga de célula de 50N, à temperatura de $23^{\circ} \mathrm{C}$ e velocidade de deslocamento de célula de $1 \mathrm{~mm} / \mathrm{s}$. Os filmes foram cortadas em formato padrão, retangular $(25 \mathrm{~mm}$ x $100 \mathrm{~mm}$ ) de acordo com a ASTM D882-12 (2012), e a distância inicial entre as garras do texturômetro foi de $50 \mathrm{~mm}$. A espessura dos filmes 
foi determinada através de micrômetro digital (Digimess, IP54).

\section{RESULTADOS E DISCUSSÃO}

Os resultados referentes ao planejamento experimental estão dispostos na Tabela 2, correspondendo aos ensaios de medida de solubilidade e das propriedades mecânicas dos filmes produzidos.

Tabela 2 - Resultados dos ensaios de solubilidade e propriedades mecânicas do planejamento

\begin{tabular}{ccccc}
\hline Ensaio & Solubilidade $\%$ & $\mathbf{E}_{\mathbf{0}}(\mathbf{M p a})$ & $\boldsymbol{\varepsilon}(\boldsymbol{\%})$ & $\boldsymbol{\sigma}(\mathbf{M p a})$ \\
\hline 1 & $20,51 \pm 0,63$ & $2306,05 \pm 144,25$ & $3,598 \pm 1,702$ & $88,36 \pm 25,16$ \\
2 & $18,19 \pm 1,19$ & $2065,72 \pm 101,34$ & $2,031 \pm 1,400$ & $51,78 \pm 19,89$ \\
3 & $17,99 \pm 2,98$ & $2393,05 \pm 137,13$ & $4,218 \pm 0,867$ & $102,58 \pm 4,16$ \\
4 & $16,47 \pm 0,31$ & $2097,22 \pm 86,63$ & $2,085 \pm 2,201$ & $48,09 \pm 24,79$ \\
5 & $20,60 \pm 0,58$ & $2663,90 \pm 170,12$ & $4,518 \pm 1,386$ & $118,16 \pm 15,58$ \\
6 & $17,02 \pm 0,28$ & $1942,95 \pm 108,19$ & $2,745 \pm 0,535$ & $64,16 \pm 4,17$ \\
7 & $19,42 \pm 0,85$ & $2294,24 \pm 120,09$ & $3,018 \pm 1,244$ & $76,12 \pm 14,99$ \\
8 & $18,00 \pm 0,93$ & $2730,52 \pm 567,28$ & $1,398 \pm 1,926$ & $48,19 \pm 39,41$ \\
9 & $18,40 \pm 0,73$ & $2461,59 \pm 99,26$ & $2,018 \pm 0,843$ & $62,16 \pm 22,27$ \\
10 & $18,79 \pm 0,80$ & $2580,53 \pm 55,49$ & $2,451 \pm 0,628$ & $78,69 \pm 14,06$ \\
11 & $17,65 \pm 1,63$ & $2165,65 \pm 246,55$ & $2,551 \pm 1,011$ & $67,07 \pm 16,21$ \\
12 & $17,05 \pm 0,98$ & $2468,50 \pm 143,96$ & $0,998 \pm 0,734$ & $38,22 \pm 18,50$ \\
\hline
\end{tabular}

Filmes padrão sem reticulação com TGase foram preparados para comparação do comportamento da reticulação. Estes resultados estão dispostos na Tabela 3.

Tabela 3 - Solubilidade e propriedades mecânicas dos filmes sem tratamento enzimático.

\begin{tabular}{ccccc}
\hline $\begin{array}{c}\text { [Gelatina] } \\
\mathbf{m g} / \mathbf{m L}\end{array}$ & Solubilidade $\%$ & $\mathbf{E}_{\mathbf{0}}(\mathbf{M p a})$ & $\boldsymbol{\varepsilon}(\boldsymbol{\%})$ & $\boldsymbol{\sigma}(\mathbf{M p a})$ \\
\hline 59,29 & $24,44 \pm 2,88^{\mathrm{a}}$ & $3011,18 \pm 46,89^{\mathrm{a}}$ & $3,965 \pm 1,797^{\mathrm{a}}$ & $101,70 \pm 25,40^{\mathrm{a}}$ \\
80 & $21,58 \pm 2,03^{\mathrm{a}}$ & $2669,35 \pm 134,22^{\mathrm{b}}$ & $5,905 \pm 0,861^{\mathrm{b}, \mathrm{a}}$ & $115,63 \pm 12,39^{\mathrm{b}, \mathrm{a}}$ \\
130 & $20,32 \pm 0,72^{\mathrm{a}}$ & $2502,45 \pm 45,59^{\mathrm{c}, \mathrm{b}}$ & $4,605 \pm 0,764^{\mathrm{c}, \mathrm{a}, \mathrm{b}}$ & $99,49 \pm 13,21^{\mathrm{c}, \mathrm{a}, \mathrm{b}}$ \\
180 & $19,57 \pm 2,49^{\mathrm{a}}$ & $2246,00 \pm 156,16^{\mathrm{d}, \mathrm{c}}$ & $1,358 \pm 1,069^{\mathrm{d}, \mathrm{a}}$ & $44,03 \pm 18,71^{\mathrm{d}}$ \\
200,71 & $19,25 \pm 1,07^{\mathrm{a}}$ & $2213,49 \pm 136,58^{\mathrm{e}, \mathrm{c}, \mathrm{d}}$ & $2,431 \pm 0,886^{\mathrm{e}, \mathrm{a}, \mathrm{c}}$ & $61,21 \pm 14,22^{\mathrm{e}, \mathrm{a}, \mathrm{c}, \mathrm{d}}$ \\
\hline \multicolumn{4}{c}{ Valores com os mesmos sobrescritos: não mostram diferença significativa a p<0,05. }
\end{tabular}

\subsection{Teste de solubilidade}

A solubilidade dos filmes tratados com TGase mostrou que baixas quantidades de enzima não tiveram efeito sobre a solubilidade, ou seja, houve pouca ou nenhuma reticulação dos filmes produzidos. Observou-se que filmes com maiores quantidades de TGase mantiveram com mais 
efetividade a conformidade estrutural da amostra, mesmo após $24 \mathrm{~h}$ de hidratação e subsequente exposição ao calor (secagem em estufa). Estudos de Kolodziejska e Piotrowska (2007) mostraram que a reticulação de filmes de gelatina com TGase não tiveram muita efetividade na redução da solubilidade quando expostos a hidratação à $100^{\circ} \mathrm{C}$, podendo indicar que os filmes com os menores percentuais de enzima não formaram ligações cruzadas o suficiente na estrutura biopolimérica.
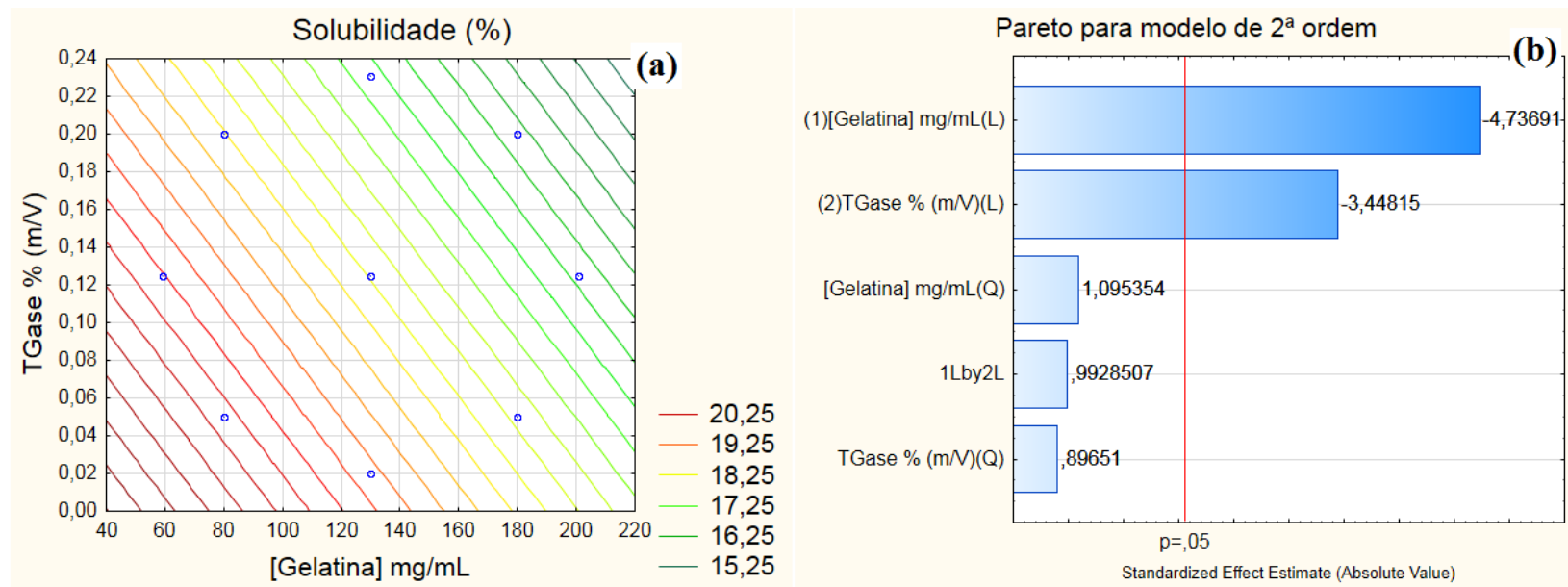

Figura 1 - Superfície de resposta (a) e gráfico de Pareto (b) para a solubilidade

Conforme Figura 1 (b), os únicos efeitos significativos no nível de significância de 5\% foram os coeficientes lineares, a concentração de gelatina e a quantidade de TGase, sendo que a interação de ambos e os coeficientes quadráticos não foram significativos. O modelo estimado foi linear (Equação 1), com baixo coeficiente de determinação $\left(\mathrm{R}^{2}\right)-50 \%$. A superfície de resposta obtida na Figura 1(a), mostra que a diminuição da solubilidade está condicionada ao aumento dos fatores estudados, o qual pode ser justificado pelo aumento de grupos amina livre em concentrações elevadas de gelatina, estes disponíveis para realização de ligações cruzadas pela ação da enzima (Macedo e Sato, 2005).

$$
\text { Solubilidade } \%=22,38-0,0218 \text {.[Gelatina] -10,59.TGase }
$$

Para os filmes sem o tratamento enzimático observou-se que não houveram diferenças significativas da variação das concentrações sobre a solubilidade das amostras (teste de Tukey a $\mathrm{p}<0,05)$. Observou-se também, por este mesmo teste (dados não mostrados aqui), que a TGase teve efeito significativo somente sobre a solubilidade, em relação aos filmes não reticulados, apenas quando usada na maior concentração estudada $(0,231 \%)$. Portanto, a enzima atuou positivamente na formação das ligações cruzadas embora não tenha sido possível detectar diferenças estatisticamente significativas.

\subsection{Propriedades mecânicas}

Os modelos encontrados para o planejamento experimental são mostrados na Tabela 4, e 
pela análise dos mesmos foi observado que a concentração de gelatina apresentou maior influência nas propriedades mecânicas dos filmes produzidos.

Tabela 4 - Modelos das propriedades mecânicas dos filmes tratados com TGase

\begin{tabular}{|c|c|c|c|c|}
\hline Resposta & Modelo & $\mathbf{p}$ & $\mathbf{R}$ & F. A. \\
\hline $\mathrm{E}_{0}(\mathrm{Mpa})$ & $2699,187^{\mathrm{a}}-(3,889 \cdot X)^{\mathrm{a}}+(1230,550 \cdot Y)^{\mathrm{b}}$ & $0,0006^{\mathrm{a}}$ & 0,610 & $0,0853^{b}$ \\
\hline$\varepsilon(\%)$ & $\begin{array}{c}9,669^{\mathrm{a}}-(0,0969 \cdot X)^{\mathrm{a}}+\left(0,0003 \cdot X^{2}\right)^{\mathrm{a}}- \\
(2,946 \cdot \mathrm{Y})^{\mathrm{b}}+\left(20,625 \cdot \mathrm{Y}^{2}\right)^{\mathrm{b}}-(0,0378 \cdot X \cdot Y)^{\mathrm{b}}\end{array}$ & $0,0130^{\mathrm{a}}$ & 0,607 & $0,5272^{b}$ \\
\hline$\sigma(\mathrm{Mpa})$ & $\begin{array}{c}193,931^{\mathrm{a}}-(1,706 . X)^{\mathrm{a}}+\left(0,006 . \mathrm{X}^{2}\right)^{\mathrm{a}}+ \\
(139,981 . Y)^{\mathrm{b}}-\left(131,507 . \mathrm{Y}^{2}\right)^{\mathrm{b}}- \\
(1,194 . X . Y)^{\mathrm{b}}\end{array}$ & $0,0003^{\mathrm{a}}$ & 0,722 & $0,5071^{\mathrm{b}}$ \\
\hline
\end{tabular}

F. A.: Falta de ajuste; ${ }^{a}$ Significativo; ${ }^{b}$ Não significativo.

$\mathrm{O}$ alongamento $(\varepsilon)$ assim como o módulo de Young $\left(\mathrm{E}_{0}\right)$ mostraram uma fraca relação linear entre as variáreis estudadas, sendo $\mathrm{R}$ de 0,607 e 0,610, respectivamente. Já a tensão máxima de ruptura $(\sigma)$ mostra clara tendência linear entre os fatores estudados, com $R$ de 0,722 .

A Figura 2(a) mostra que o módulo de Young atinge um máximo quando há um aumento da quantidade de enzima para valores próximos a $0,2 \%$ e para baixas concentrações de gelatina; assim, pode-se afirmar que a reticulação da proteína infere rigidez ao filme. $\mathrm{O}$ fato de valores altos de concentração de gelatina tenderem a valores inferiores de $\mathrm{E}_{0}$, pode ser decorrente do aumento da espessura do filme devido a altas concentrações de gelatina, interferindo na determinação do mesmo (Huang et al., 2004). Entretanto, observou-se por teste de Tukey (dados não exibidos aqui) que as propriedades mecânicas foram pouco afetadas pela adição de enzima, sendo que houve apenas uma redução significativa do $E_{0}$ para concentração de $80 \mathrm{mg} / \mathrm{mL}$ com $0,5 \%$ de TGase comparado ao filme de referência B $(80 \mathrm{mg} / \mathrm{mL}$ de gelatina sem enzima); para o alongamento houve redução significativa quando utilizado nível máximo de enzima $(0,231 \%)$; quanto à tensão máxima de ruptura, foi observada uma redução estatisticamente não significativa em comparação aos filmes não tratados enzimaticamente.

A redução do alongamento para os filmes tratados enzimaticamente quando em comparação aos filmes não tratados (Tabela 3), pode ser decorrente da diminuição da mobilidade devido à reticulação das cadeias poliméricas do filme (Carvalho e Grosso, 2006). Observa-se pelo gráfico de superfície de resposta (Figura 2 (b)), que o aumento da concentração de gelatina e da quantidade de enzima resultam na diminuição do alongamento, sendo este efeito mais predominante em regiões de concentrações mais altas de gelatina, pois para valores abaixo de 120 $\mathrm{mg} / \mathrm{mL}$ de gelatina tem-se pouca influência da transglutaminase. Este comportamento também é visível sobre a tensão de ruptura (Figura 2 (c)). Entretanto, conforme Piotrowska et al. (2008), o tratamento com a TGase confere fragilidade ao filme, ocasionando assim a redução do alongamento bem como a diminuição da tensão máxima de ruptura, apesar de que pode se 


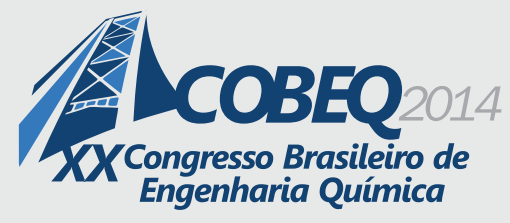

observar que a concentração de gelatina foi o fator significativo (estatisticamente) na variação das respostas estudadas.

Babin e Dickinson (2001) observaram que o tratamento com TGase em soluções de gelatina do tipo A e B podem apresentar diferentes efeitos na flexibilidade da cadeia de gelatina, dependendo se a reticulação covalente ocorre predominantemente antes ou depois da formação de zonas induzidas por arrefecimento. Portanto, observaram que o tratamento enzimático faz com que o desenvolvimento de uma rede de percolação polimérica com propriedades elásticas fique inibida, devido a formação de ligações cruzadas covalentes intra e intermolecular na solução de gelatina.
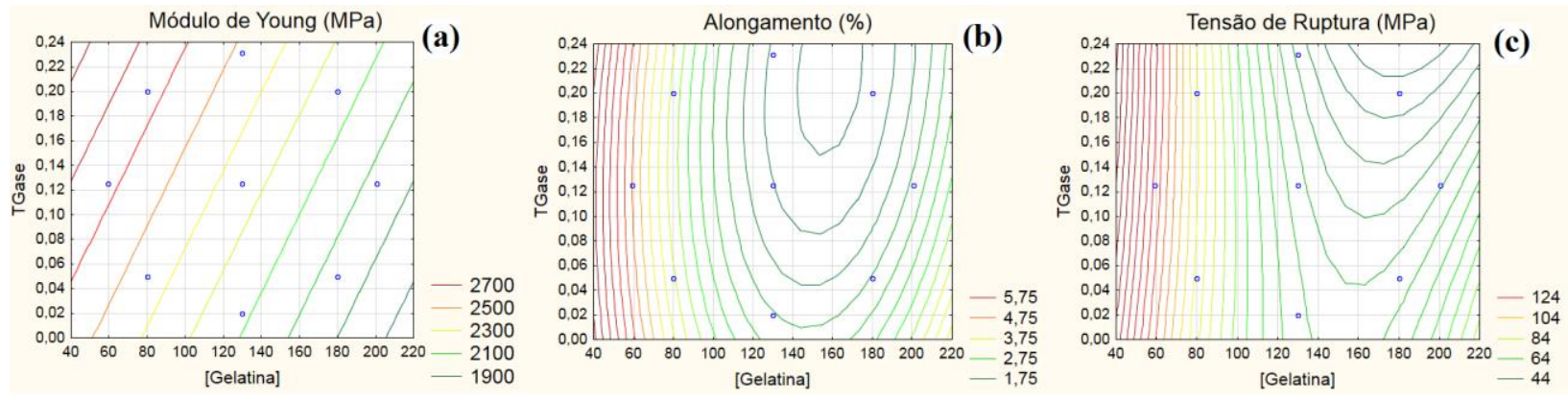

Figura 2 - Superfícies de resposta para o módulo de Young (a), alongamento (b) e tensão de ruptura (c)

\section{CONCLUSÕES}

A solubilidade dos filmes tratados enzimaticamente foi reduzida devido ao efeito reticulante da TGase, sendo que a enzima teve ação significativa em seu nível máximo $(0,231 \%)$ comparativamente aos filmes não tratados. No entanto, a variação do teor de TGase não interferiu significativamente na solubilização destes filmes, porém contribuiram para a manutenção da integridade da amostra. As propriedades mecânicas foram modificadas devido à criação de ligações cruzadas na rede proteica, sendo que tanto o alongamento quanto a tensão de ruptura foram reduzidos, ou seja, o tratamento enzimático conferiu fragilidade à estrutura concomitantemente a um aumento da rigidez (módulo de Young) dentro dos níveis do planejamento, apesar da diferença entre os ensaios não ter sido significativa. No entanto, quando os dados dos filmes com e sem ação da enzima foram comparados, observou-se redução nos valores das propriedades mecânicas, com pouca diferença estatisticamente significativa. Portanto, a enzima TGase apresentou efeito reticulante significativo apenas quando utilizada em quantidade acima de $0,2 \%$.

\section{REFERÊNCIAS}

ASTM-D882-12. Standard Test Method for TensileProperties of Thin Plastic Sheeting. Book of Standards.: ASTM International, v. 08.01, 2012.

BABIN, H.; DICKINSON, E. Influence of transglutaminase treatment on the 
thermoreversible gelation of gelatin. Food Hydroc., v. 15, p. 271-276, 2001.

BALDWIN, E. A.; NISPEROS - CARRIEDO, M. O.; BAKER, R. A. Use of edible coatings to preserve quality of lightly (and slightly) processed products. Crit. Rev. F. Scien. Nutrit., v. 35, n. 6, p. 509-524, 1995.

BHATTACHARYA, A.; RAWLINS, J. W.; RAY, P. Polymer Grafting and Crosslinking. Wiley, 2008

CANEVAROLO, S. V. Ciências dos polímeros: um texto básico para tecnólogos e engenheiros. Artliber, 2006.

CARVALHO, R. A. D.; GROSSO, C. R. F. Efeito do tratamento térmico e enzimático nas propriedades de filmes de gelatina. Food Science Technol. (Campinas), v. 26, p. 495-501, 2006.

CUQ, B. et al. Selected Functional Properties of Fish Myofibrillar Protein-Based Films As Affected by Hydrophilic Plasticizers. J. Agric. Food Chem., v. 45, n. 3, p. 622-626, 1997.

EBNESAJJAD, S. Handbook of Biopolymers and Biodegradable Plastics: Properties, Processing and Applications. Elsevier Science, 2012.

HUANG, Z. M. et al. Electrospinning and mechanical characterization of gelatin nanofibers. Pol., v. 45, n. 15, p. 5361-5368, 2004.

KOŁODZIEJSKA, I.; PIOTROWSKA, B. The water vapour permeability, mechanical properties and solubility of fish gelatin-chitosan films modified with transglutaminase or 1-ethyl-3(3-dimethylaminopropyl) carbodiimide (EDC) and plasticized with glycerol. Food Chem., v. 103, n. 2, p. 295-300, 2007.

MACEDO, J. A.; SATO, H. H. Propriedades e aplicações da transglutaminase microbiana em alimentos. Alim. Nut. Araraq., v. 16, 2005.

NUSSINOVITCH, A. CHAPTER 10 - Biopolymer Films and Composite Coatings. Modern Biopolymer Science. San Diego: Academic Press, 2009.

PIOTROWSKA, B. et al. Influence of transglutaminase or 1-ethyl-3-(3-dimethylaminopropyl) carbodiimide (EDC) on the properties of fish-skin gelatin films. Food Hydroc., v. 22, n. 7, p. 1362-1371, 2008.

SALDIVAR-GUERRA, E.; VIVALDO-LIMA, E. Handbook of Polymer Synthesis, Characterization, and Processing. Wiley, 2013.

\section{AGRADECIMENTOS}

À Central de Análise EQA - UFSC e à CAPES. 